\title{
A study of early versus conventional oral feeding in women undergoing caesarean section
}

\author{
Devina Nagraj*, Ching Ling Yi, Asha R. Dalal
}

Department of Obstetrics and Gynaecology, TNMC and BYL Nair Hospital, Mumbai, Maharashtra, India

Received: 23 September 2020

Revised: 27 October 2020

Accepted: 28 October 2020

\author{
*Correspondence: \\ Dr. Devina Nagraj, \\ E-mail: dvna1008@gmail.com
}

Copyright: () the author(s), publisher and licensee Medip Academy. This is an open-access article distributed under the terms of the Creative Commons Attribution Non-Commercial License, which permits unrestricted non-commercial use, distribution, and reproduction in any medium, provided the original work is properly cited.

\begin{abstract}
Background: Traditionally women undergoing lower segment caesarean section (LSCS) had oral food withheld for12-24 hours to prevent gastrointestinal complications. However, it was observed in many studies, that early oral feeding to patients post LSCS improves recovery and decreases chances of post-operative complications.

Methods: This was a randomized interventional study conducted in a public hospital. Uncomplicated patients who underwent LSCS under regional anesthesia were selected. Group A and B with 100 participants each were made. One group (A) was given early orals (6 hours) and other group (B) was given orals after 12 hours.

Results: The early feeding group had earlier return of bowel movements and earlier passage of flatus ( 8 and 13 hours) as opposed to delayed feeding group (10 and 18 hours). 13\% patients in the early feeding group had complications as opposed to $43 \%$ patients in the delayed feeding group. The most common complication was abdominal distension which was seen in 5 and 10 patients of the early and delayed feeding group respectively.

Conclusions: Early oral feeding after LSCS can lead to early recovery, better mother and child health, better utilization of the hospital resources and decreased financial burden on the family as well as the government in the long run.
\end{abstract}

Keywords: Early oral feeding, Caesarean section, Randomized interventional study, Post-operative recovery

\section{INTRODUCTION}

After general abdominal surgery, it is customary for the patient to take no fluid or food by mouth for a specific period, or until the return of bowel function as evidenced by propulsive bowel sounds or passing of flatus or stool. Abdominal surgery abolishes normal bowel motility immediately post operatively and the onset of bowel function is influenced by the type of surgery performed; and that there may be many factors contributing to paralytic ileus (decreased or absence of intestinal peristalsis following abdominal surgery characterized by abdominal tenderness and distension, absence of bowel sounds, lack of flatus and by nausea and vomiting) other than early feeding, such as neural and hormonal factors, involvement of the sympathetic and parasympathetic nervous system, use of narcotics and the type of anesthetic agents used. ${ }^{1}$

Traditionally, women who had a caesarean section had oral food withheld for the first 12-24 hours in the belief that this would prevent gastrointestinal complications. After caesarean section, practices vary considerably between institutions and individual practitioners, ranging from early oral feeds to delayed introduction of feeds which may be after 12-24 hrs. or more. The practice of allowing early oral fluids and food after caesarean section is often based on assumption that the bowels are not usually exposed or handled during caesarean section and peritoneal irritation occurs significantly less so one would therefore not expect bowel function to be disturbed. The main purpose of traditional dietary regimen is to prevent the occurrence of ileus after an abdominal surgery. ${ }^{1}$ 
Oral feeding was started only after the bowel sounds were heard and patient had passed flatus. It was believed that the bowels need rest after all abdominal surgeries, and feeding will interfere with the function of resting bowels. This belief was not only prevalent among the lay public but even the medical staff felt the same. ${ }^{2}$

A meta-analysis in 2002 revealed no evidence to indicate that early oral feeding after caesarean delivery increases the chances of ileus or other post-operative complications; rather it has been reported to decrease post-operative metabolic stress response, regress ileus symptoms and lower the morbidity rates. ${ }^{1}$

Another recent meta-analysis of studies comparing early oral feeding with delayed oral feeding after CS found out that early oral feeding after CS enhances return to bowel function and does not increase the risk of postoperative complications. ${ }^{3}$

It has been observed in a number of studies that early orals to mother post-surgery led to early mobilization, recovery and hence early discharge. This decreases chance of post-operative complications and reduce the cost of post-operative stay. Thus, it leads to decreased financial burden on the family and government as well as efficient utilization of hospital resources and manpower. This was the motivation for the study with the aim to evaluate safety a and efficacy of early oral feeding (6 hours) as compared to conventional feeding practices (12 hours) in post lower segment caesarean section patients.

\section{METHODS}

\section{Study type}

A randomized interventional study.

\section{Study site}

Department of Obstetrics and Gynecology, Topiwala National Medical College and BYL Nair Hospital, Mumbai, Maharashtra, India

\section{Study period}

July 2017 - June 2018.

\section{Sample size}

It was planned to enroll 100 cases per group i.e. total 200 cases for the study.

\section{Inclusion criteria}

All post-natal mothers undergoing LSCS at>38 weeks without any complications like PIH (pregnancy induced hypertension), diabetes, anemia, ante partum hemorrhage, post-partum hemorrhage between age of 18-35 years were included in the study.

\section{Exclusion criteria}

Exclusion criteria were women receiving general anesthesia, women with caesarean section associated with any complications like anemia, bleeding or other systemic diseases like diabetes mellitus, thyroid dysfunction, heart and kidney disorders, pre-eclampsia, eclampsia post-partum hemorrhage requirement of blood and blood products DIC and elevated risk placental anomalies, women undergoing operative complications, bladder injury, excessive bleeding $>1000 \mathrm{ml}$, bowel injuries

\section{Ethical clearance}

The ethical clearance was obtained from the institutional ethical committee of the institution- Topiwala National Medical College and BYL Nair Hospital, Mumbai, after submitting the protocol and subsequently answering all their queries.

\section{Study procedure}

Consent of the patient was taken prior to the caesarean section. Patients randomly allocated to group A and group B. Randomization is done as every alternate patient is allocated into 2 groups. Patients are labelled 1,2,1,2 and so on with every 1 going to study group of early feeding (group A) and 2 going to control group of conventional feeding (group B).

Group A patients started with oral sips of water $6 \mathrm{hrs}$. post operatively followed by liquid diet and soft diet according to patient tolerance.

Group B started on oral sips $>12$ hrs. post-delivery followed by liquid diet and soft diet as per patient tolerance.

Parameters like nausea, vomiting other gastrointestinal symptoms, time to first passage of flatus, time to presence of bowel sounds, time to passage of stools, duration of IV fluids, duration of hospital stay, were studied. Moreover, post-operative pain in terms of visual analog scale, complications like ileus, wound infection, fever, abdominal distension, nausea and vomiting were studied in both groups.

The time of onset of surgery was designated as zero hour. The day of surgery (day 1) was considered to be the first 24 hours, the second postoperative day (day 2) encompassed the next 24-48 hours and the third postoperative day (day 3 ) covered the next 48-72 hours. The operative time was defined as the time from the onset of surgery to the completion of skin closure. The duration of intravenous fluid administration was defined as the time from the onset of surgery to the time of stopping IV fluids. Intravenous fluids were stopped when patients were capable of consuming adequate solid and liquid diet. The pain scores were assessed based on VAS (visual analog scale) on day 3 of LSCS in all patients. 
Time interval for bowel movements to appear was defined as the time from the onset of surgery until the first detection of active bowel sounds. Ileus symptoms included symptoms of anorexia, abdominal cramping, non-persistent nausea and vomiting as well as mild abdominal distension on physical examination. Other symptoms included marked abdominal distension with more than three episodes of vomiting in a 24-hour period or an inability to tolerate oral liquids and a need to delay the stepped-up diet.

The length of hospital stay was counted from the day of surgery to the day the patients were allowed to be discharged from the hospital and signed by the physician under the conditions that they were able to tolerate a regular diet without emesis, passed flatus or had a bowel movement, and demonstrated no febrile morbidity for at least 24 hours. Postoperative febrile morbidity was considered to be an oral temperature equal to or exceeding $38^{\circ} \mathrm{C}$ or $100.4^{\circ} \mathrm{F}$ on 2 or more occasions, at least 6 hours apart, occurring greater than 24 hours.

After obtaining the written inform consent in vernacular languages, pre-tested semi-structured proforma prepared in accordance with the study objectives was used for collecting information about the respondent. Face to face interview of the patients was conducted. Time taken for questionnaire filling was 20-25 minutes for each person. Labor records were noted. Patient were followed till day of suture removal.

\section{Statistical analysis}

Statistical analysis was carried out with the help of SPSS (version 20) for Windows package (SPSS Science, Chicago, IL, USA). Quantitative data like time interval for bowel movements to appear, duration of IV fluids administration, length of hospital stay is represented using median and IQR (inter quartile range) qualitative data represented in the form of frequency and percentage. Association between qualitative variables like occurrence of paralytic ileus, occurrence of other complications assessed by chi-square test for all $2 \times 2$ tables.

\section{RESULTS}

The median age of women of early oral fluids group is 26 years whereas that of late feeding group is 25 years. Majority of women in the present study were from primigravida $37.5 \%$ (75) followed by 2nd (28.5\%) and 3rd $(23.5 \%)$ gravida respectively. The median gestational age was 39.1 weeks as per the earliest USG scan report.

Most common indication in both early feeding and conventional feeding group was previous LSCS, 20 in group $\mathrm{A}$ and 16 in $\mathrm{B}$ followed by meconium stained liquor seen in 17 in group A and 12 in group B. This was followed by fetal distress in 8 in group $A$ and 12 in group $\mathrm{B}$ and oligohydramnios in 8 women in both group A and B. Table 1 shows the result of variables studied in the group $\mathrm{A}$ and $\mathrm{B}$.

The complications were seen less in the early feeding group, $13 \%$ as opposed to $43 \%$ patients in the delayed feeding group. Ileus was seen in 1 patient in early feeding group as opposed to 7 patients in delayed feeding group. The most common complication was abdominal distension which was seen in 6 patients of the early feeding group as opposed to 14 patients in the delayed feeding group. Table 2 demonstrates the different postoperative complications seen in early and conventional feeding groups. The median duration of LSCS was 72.5 mins with maximum duration of 180 mins and minimum duration of 45 mins. $53 \%$ of the study participants received IV fluids for 24 hours. $41.5 \%$ (83) women started breastfeed within 1 hour after LSCS followed by $37 \%$ (74) women started breastfeeding within $2 \mathrm{hrs}$.

Table 1: Variables studied vs oral feeding.

\begin{tabular}{|lllll|}
\hline \hline & $\begin{array}{l}\text { Early feeding (6 } \\
\text { hours) median (IQR) }\end{array}$ & $\begin{array}{l}\text { Delayed feeding } \\
(\mathbf{1 2} \text { hours) median } \\
\text { (IQR) }\end{array}$ & P value & Result \\
\hline Age (years) & $26(5)$ & $25(6)$ & 0.3023 & Not significant \\
\hline Nulliparity & $42.7 \%$ & $57.3 \%$ & 0.0460 & Significant \\
\hline Duration of hospital stay (days) & $5(2)$ & $6(3)$ & 0.0000 & Highly significant \\
\hline Return of bowel movement (hours) & $8(3)$ & $10(4)$ & 0.0153 & Significant \\
\hline Passage of flatus (hours) & $13(4)$ & $18(5)$ & 0.0000 & Highly significant \\
\hline Duration of IV fluid (hours) & $20(6)$ & $24(0)$ & 0.0000 & Highly significant \\
\hline Amount of IV fluid (m) & $2000(500)$ & $2500(500)$ & 0.0000 & Highly significant \\
\hline Time to mobilization (hours) & $16(4)$ & $20(4)$ & 0.0000 & Highly significant \\
\hline Removal of foleys catheter (hours) & $16(3)$ & $24(5)$ & 0.0000 & Highly significant \\
\hline First defecation (hours) & $36(15)$ & $48(32)$ & 0.0000 & Highly significant \\
\hline Pain score & $3(1)$ & $3(2)$ & 0.0015 & Significant \\
\hline
\end{tabular}

(As the data was not following normality so median was considered. Shapiro-Wilk normality test shows data is non-normal, so Mann Whitney test is used.) 
Table 2: Post-operative complications vs oral feeding.

\begin{tabular}{|c|c|c|c|c|}
\hline \multirow{2}{*}{ Complications } & & \multicolumn{2}{|l|}{ Oral feeding } & \multirow{2}{*}{ Total } \\
\hline & & 6 hours (A) & 12 hours (B) & \\
\hline \multirow{2}{*}{ Abdominal distension } & No. of cases & 6 & 14 & 20 \\
\hline & $\%$ Within complication & 30 & 70 & 100.0 \\
\hline \multirow{2}{*}{ Diarrhoea } & No. of cases & 0 & 3 & 3 \\
\hline & $\%$ Within complication & 0.0 & 100.0 & 100.0 \\
\hline \multirow{2}{*}{ Fever } & No. of cases & 2 & 8 & 10 \\
\hline & $\%$ Within complication & 20.0 & 80.0 & 100.0 \\
\hline \multirow{2}{*}{ Ileus } & No. of cases & 1 & 7 & 8 \\
\hline & $\%$ Within complication & 12.5 & 87.5 & 100.0 \\
\hline \multirow{2}{*}{ Nausea and vomiting } & No. of cases & 3 & 4 & 7 \\
\hline & $\%$ Within complication & 42.9 & 57.1 & 100.0 \\
\hline \multirow{2}{*}{ No } & No. of cases & 87 & 57 & 144 \\
\hline & $\%$ Within complication & 60.4 & 39.6 & 100.0 \\
\hline \multirow{2}{*}{ wound infection } & No. of cases & 1 & 7 & 8 \\
\hline & $\%$ Within complication & 12.5 & 87.5 & 100.0 \\
\hline \multirow{2}{*}{ Total } & No. of cases & 100 & 100 & 200 \\
\hline & $\%$ Within complication & 50.0 & 50.0 & 100.0 \\
\hline
\end{tabular}

\section{DISCUSSION}

The median gestational age was 39.1 weeks as per the earliest USG scan report. The maximum and minimum gestational age was 42 and 38 weeks respectively. Nantasupha et al found the mean gestational age in their study as $38.6 \pm 0.6$ and $38.6 \pm 0.7$ weeks by dates and earlier USG scan in early and late oral feeding groups. ${ }^{4}$ In another study done by Mehta et al it was found to be $38.7 \pm 1.3$ and $38.5 \pm 1.1$ by dates and earlier USG scan respectively in both groups respectively and hence similar results were found. ${ }^{5}$

Most common indication in both the groups was previous LSCS followed by meconium stained liquor, fetal distress and oligohydramnios. In the current study 20 and 16 had previous LSCS as opposed 20.8 and $31.9 \%$ in study by Ogbadua et al and $10-12 \%$ in the study by Mehta et al., The most common cause of LSCS in study by Ogbadua et al was CPD and Mehta et al was failure to progress of labor. The results of the current study were not similar to the other studies but it was observed that previous LSCS was one of the most common indications in most of the studies. However, there was no significant association between indications of LSCS and oral feeding.

The median hospital stay in the current study in early and delayed feeding groups was 5 days and 6 days respectively which was found to be statistically significant. In the study done by Ogbadua et al the duration of hospital stay was $4.2 \pm 0.7$ and $4.2 \pm 1.2$ days in early and late feeding groups respectively. ${ }^{6}$ In the study done by Devi et al the duration of hospital stay was $4.66 \pm 0.87$ and $6.68 \pm 1.04$ days in early and late feeding groups respectively.7 The results of the current study were similar to that of the study by Devi et al. ${ }^{7}$
The median time for bowel movements to appear in the early and delayed feeding groups was 8 hours and 10 hours which was found to be significant statistically. As opposed to this in the study done by Devi et al the bowel movements appeared in $6.97 \pm 0.71$ and $14.96 \pm 4.97 \mathrm{hrs}$. in early and late feeding groups respectively. ${ }^{7}$ In the study done by Mehta et al the bowel movements appeared in $7.8 \pm 1.4$ and $14.7 \pm 2.1 \mathrm{hrs}$ in early and late feeding groups respectively. ${ }^{5}$ In all it was seen that bowel movements appeared early in early feeding group than those on conventional feeding.

In the group with early and delayed oral feeding the median duration of IV fluids given was 20 hours and 24 hours respectively. In the study done by Ogbadua et al the duration of IV fluids given was 18.9 \pm 1.7 and $25.0 \pm 2.4$ days in early and late feeding groups respectively which was significant. ${ }^{6}$ In another study done Devi et al the duration of IV fluids given was $23.4 \pm 2.97$ and $40.39 \pm 4.78$ hrs. $^{7}$ In early and late feeding groups respectively which was significant. In the study done by Nantasupha et al the duration of IV fluids given was $14.8 \pm 4.6$ and $27.7 \pm 5.5$ days in early and late feeding groups respectively which was significant by Chi-square test. ${ }^{4}$ The results of the current study were found to be similar to that of the study by Ogabadua et al. ${ }^{6}$

In the early and conventional feeding group the median amount of IV fluids given was in $2000 \mathrm{ml}$ and $2500 \mathrm{ml}$ respectively which was highly significant. Whereas in the study done by Nantasupha et al the amount of IV fluids (in liters) given was $1.1 \pm 0.3$ and $2.8 \pm 0.5$ liters in early and late feeding groups respectively which was significant by Chi-square test. ${ }^{4}$ Also, in another study done by Kathpalia the amount of IV fluids given was $4.2 \pm 1.2$ and $6.1 \pm 0.8$ bottles per patient in early and late feeding groups respectively which was significant. ${ }^{2}$ In 
another study done Mehta et al the amount of IV fluids given was $3.9 \pm 0.9$ and $7.7 \pm 0.7$ bottles per patient in early and late feeding groups respectively which was significant. $^{5}$

In the early feeding and conventional (delayed) feeding group the median time required to mobilize the patient after CS was $16 \mathrm{hrs}$. and 20 hours respectively which was highly significant. Similar results were seen in study done Kathpalia the average time of ambulation was $16.3 \pm 1.7$ and $22.5 \pm 1.7 \mathrm{hrs}^{2}$ in early and late feeding groups respectively which was significant. Also, In the study done by Nantasupha et al the time required to mobilize the patient after CS (in hrs.) was 20.3 \pm 3.6 hrs. and $30.0 \pm 4.4$ in early and late feeding groups respectively (statistically significant). ${ }^{4}$

In the early feeding group and late feeding group the median time required for removal of Foley's Catheter was $16 \mathrm{hrs}$. and 24 hours respectively which was statistically significant. Similarly, in study done Ogbadua et al the median time required for removal of Foley's catheter (in hrs.) was 18.3 and 20.9 hrs in early and late feeding groups respectively which was statistically significant. ${ }^{6}$ Also, Nantasupha et al in their study found that the average time required for the removal of Foley's catheter was $18.3 \pm 3.6$ and $28.2 \pm 4.2 \mathrm{hrs}$. in early and late feeding groups respectively. 4

There is highly significant statistical association between time required for first passing of stool after CS (in hrs.) and oral feeding by Mann Whitney test. In the group in which orals are given after 6 hours, the median time required for first passing of stool after CS (in hrs.) was 36 hrs. with inter quartile range (IQR) of $15 \mathrm{hrs}$. whereas in the group in which orals are given after $12 \mathrm{hrs}$. the median time required for first passing of stool after CS (in hrs.) was $48 \mathrm{hrs}$. with inter quartile range (IQR) of 32 hrs. In the study done by Mehta et al average time required for first passing of stool after CS was 42.3 \pm 6.7 and $69.4 \pm 7.3 \mathrm{hrs}$ in early and late feeding groups respectively which was significant by Chi-square test. ${ }^{5}$ In all it was observed that the time required for first passing of stool after CS (in hrs.) was higher in conventional feeding group as compared to early feeding group.

Although $72 \%$ (144) of study participants did not have any complications amongst those who had complications majority of them belonged to the group to which orals were given after 12 hours. Among the complications, abdominal distension (10\%) was most common followed by fever $(5 \%)$, ileus (4\%) and diarrhea in $(1.5 \%)$. Ileus one of the dangerous complications was seen in 7 patients in delayed feeding group as opposed to 1 in early feeding group. In study done by Mehta et al the mild ileus symptoms were there in $7 \%$ and $9 \%$ in early and conventional feeding groups. ${ }^{5}$

\section{Limitations}

Limitation of the study was that as the sample size of the study was very small so the results not necessarily reflect to the large population. Also, this study was restricted to uncomplicated and low risk LSCS.

\section{CONCLUSION}

The study showed that early feeding had a lot of advantages over the conventional group. The patients in whom early feeding was started had earlier return of bowel functions, early ambulation, less chance of complications and early discharge from hospital. Furthermore, this led to better utilization of hospital resources and decreased financial load on the family as well as on the government in the long run. These findings add to the already robust literature on the safety and benefits of early oral feeding after uncomplicated low risk LSCS. It can be concluded that there is enough evidence for clinicians worldwide to safely implement early oral feeding following LSCS especially in low risk and otherwise healthy mothers.

\section{ACKNOWLEDGMENTS}

Special thanks to the Head of the Department, Department of Obstetrics and Gynecology at BYL Nair Hospital to give me this opportunity and support to conduct the study.

\section{Funding: No funding sources}

Conflict of interest: None declared

Ethical approval: The study was approved by the Institutional Ethics Committee

\section{REFERENCES}

1. Mangesi L, Hofmeyr GJ. Early compared with delayed oral fluids and food after caesarean section. Cochr Databa Systemat Review. 2002;3:CD003516.

2. Kathpalia SK. Early maternal feeding versus traditional delayed feeding after cesarean section: a pilot study. J Obstet Gynecol Ind. 2017;67(3):17882.

3. Huang $\mathrm{H}$, Wang $\mathrm{H}, \mathrm{He}$ M. Early oral feeding compared with delayed oral feeding after cesarean section: A meta-analysis. J Matern Fetal Neonatal Med. 2016;29:423-9.

4. Nantasupha C, Ruengkhachorn I, Ruangvutilert P. Effect of conventional diet schedule, early feeding and early feeding plus domperidone on postcesarean diet tolerance: A randomized controlled trial. J Obstet Gynaecol Resear. 2016;42(5):519-25.

5. Mehta S, Gupta S, Goel N. Postoperative oral feeding after cesarean section-early versus late initiation: a prospective randomized trial. J Gynecolo Surg. 2010;26(4):247-50.

6. Ogbadua AO, Agida TE, Akaba GO, Akitoye OA, Ekele BA. Early versus delayed oral feeding after 
uncomplicated cesarean section under spinal anesthesia: A randomized controlled trial. Niger $\mathbf{J}$ Surg. 2018;24(1):6-11.

7. Devi SS, Pillai SK, Vijayaraghavan J. A Comparative Study of Early versus Conventional delay in Postoperative oral intake in Women undergoing Cesarean Section under regional anaesthesia. Ind J Obstet Gynaecol Res. 2015;2(4):276-82.

Cite this article as: Nagraj D, Yi CL, Dalal AR. A study of early versus conventional oral feeding in women undergoing caesarean section. Int J Reprod Contracept Obstet Gynecol 2020;9:4839-44. 